\title{
Modeling and Simulation of HVDC Transmission Based on PSCAD
}

\author{
Tie Ma \\ North China Electric Power University Beijing 102200010 \\ Tonymmbingo@126.com
}

Keywords: PSCAD; Simulation; HVDC; Modeling; Converter

\begin{abstract}
With the continuous development of DC transmission technology, more and more high-voltage UHV project put into operation. Therefore, we need a more reliable and flexible way to participate in the design inspection work. In this paper, we first analyze the main components of HVDC transmission system components, including commutation transformers, filters, 12 pulsating converters, etc., and established a model combined with its electrical principle characteristics. Secondly, we based on PSCAD / EMTDC simulation software to join the HVDC transmission lines, and through the relevant reference data for its adjustment parameters. Finally, we simulated the waveform in the case of normal start and short circuit fault, analyzing the waveform and calculating the comparison.
\end{abstract}

\section{Basic Concepts of HVDC}

HVDC transmission is defined as the use of stable DC, which reacts almost 0 , no synchronization problems such as the use of high-power long-distance DC transmission. The transmission current is transported from one end to the other in the form of DC to realize long-distance and large-scale energy transportation. It has the advantages of improving the stability of the AC system, realizing the asynchronous network of the $\mathrm{AC}$ system, and transmitting the power higher under the same voltage level. The main form is AC-DC-AC conversion. Typical high-voltage DC form includes bipolar two-terminal DC transmission system, the same pole at both ends of the DC transmission system, back-to-back DC transmission system, multi-terminal DC transmission system.

\section{Development of HVDC Technology}

DC transmission technology has been applied since the 1950s and has been developed rapidly in recent years. According to incomplete statistics, there are nearly 100 HVDC projects in the world, covering more than 20 countries on five continents. It cooperates with $\mathrm{AC}$ transmission to form a modern power transmission system.

China's high-voltage DC transmission research started late, from the early 60s, in 1987, China's Zhoushan in Zhejiang put into operation the first independent design, construction, equipment, localization of the line. After more than 10 years of development, in 2001 the Southern Power Grid put into Tianshengqiao to Guangzhou DC transmission system, the working voltage of $500 \mathrm{kV}$, the capacity of $1800 \mathrm{MW}$. With the continuous improvement of China's scientific and technological level, in 2010, the first UHVDC pilot project of the national grid, Xiangjiaba - Shanghai $\pm 800 \mathrm{kV}$ grade UHVDC transmission project built and put into operation. Based on China's energy demand and uneven distribution of the urgent reality, as well as the broad masses of scientific and technological workers to study, China will come from behind, will lead the world in the near future to achieve high voltage $\pm 1100 \mathrm{kV}$ line construction!

In order to better study the structural characteristics of HVDC transmission and to understand the relevant rules of its output waveform, we use the learned knowledge to consult the relevant information on the traditional HVDC transmission model, and according to the reference and their own experience to adjust the corresponding the specific parameters. Finally we will use the PSCAD method for its simulation analysis. 


\section{Main Power Equipment Model Established}

We first take the traditional unipolar HVDC as an example. To simplify the line model, we can generalize it as AC voltage sources, voltage and current transformers, AC transformers, AC filters, 12-pulsed converters, a transmission wire, etc. The AC voltage through the rectification and inverter achieve the ultimate energy transmission, followed by the various components for each one.

AC Voltage Source. As the AC voltage source contains a certain internal resistance and inductance, we choose an ideal AC voltage and resistance and capacitance in parallel after the series to simulate the equivalent, as shown below

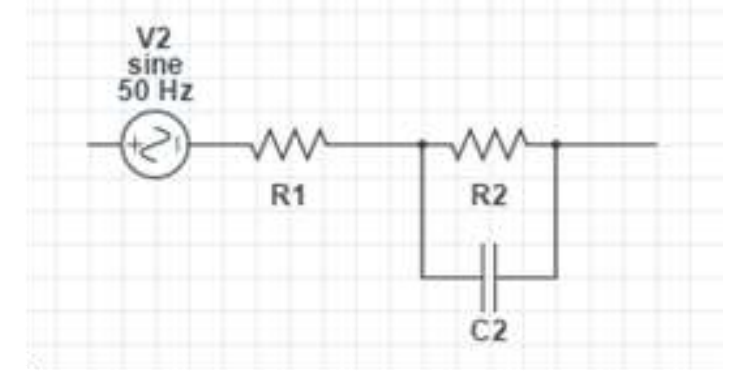

Figure 1. AC voltage source equivalent model

Voltage and Current Transformers. In order to measure the current and voltage on both sides of the transmission line, we choose to install current and voltage transformers at both ends of the line for easy design and simulation. We approximate the ideal measurement device.

Converter Transformer. The converter valve provides the appropriate level of ungrounded three-phase voltage source. As the transformer valve side is not grounded, the DC system can establish its own ground reference point, usually the valve converter positive or negative ground. Through a group into $\mathrm{Y}-\mathrm{Y}$, the other group connected to $\mathrm{Y}$-, respectively, for a commutation bridge power supply. The three-phase voltage supplied to a commutation bridge and the three-phase voltage difference 30 provided to the other bridge. The AC current waveform of the two bridges is added so that the waveform is closer to the sine wave than the AC current waveform of each 6-pulse bridge, eliminating the ripple reduction of the 5, 7th harmonic output DC voltage, then the voltage ripple is reduced and the parameters corresponding to the electrical diagram as follows,

AC Filter. In order to achieve the requirements of reactive power capacity and filtering, designers often use single-tuned filter, dual-tuned filter, three-tuned filter and second-order high-pass filter in HVDC transmission, as shown in the following figure. Through the RLC and other components in series and parallel combination, in order to achieve the compensation for the local reactive power to achieve the voltage rise, while the electrical parameters of the reasonable set to achieve a specific

frequency of current filtering, so that the introduction of the earth. According to the actual situation and the introduction of relevant literature, we mainly used in the pscad simulation of the following 


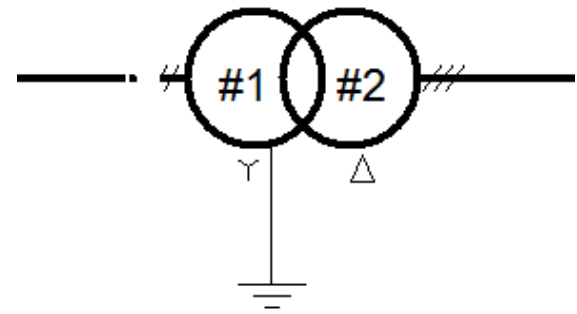

Figure 2. Converter transformer

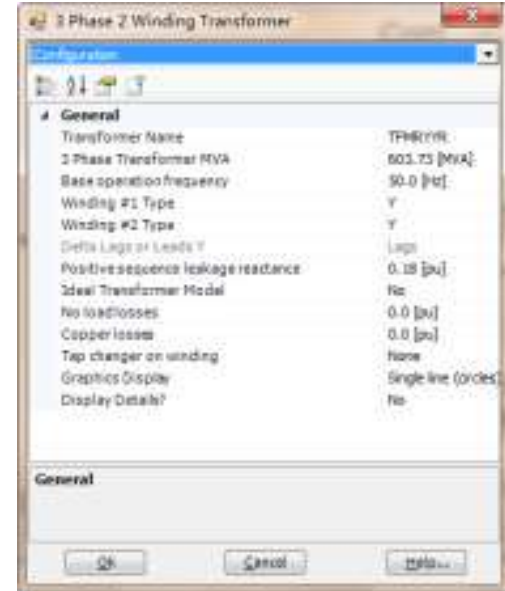

Figure 3. Corresponding parameters

second-order high-pass filter and C-type damping filter, the circuit structure as shown Corresponding value is based on the reference values described in the references.

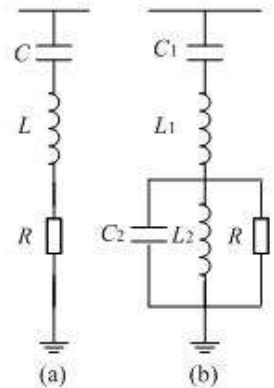

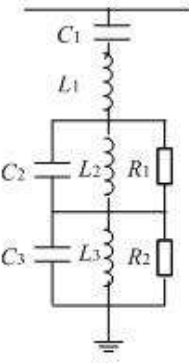

(c)
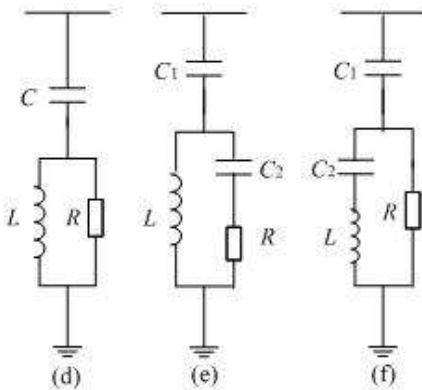

Figure 4. Typical filter structure

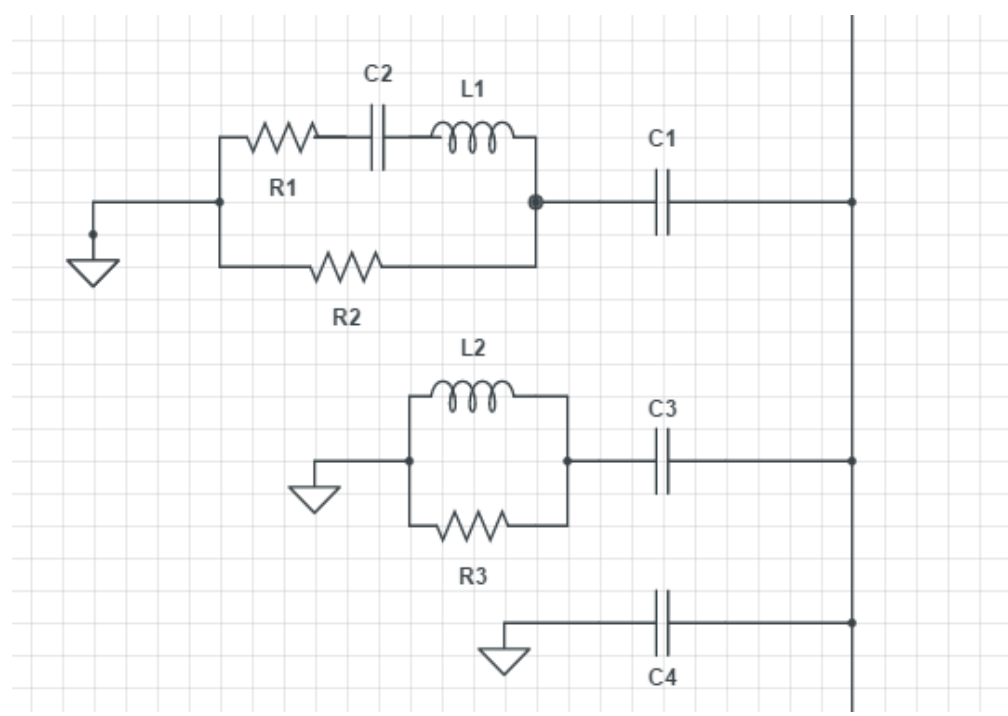

Figure 5. The filter structure of the model

12 Pulsating Converter. As a traditional high-voltage DC transmission in the core device, 12 pulsating converter has a more complex structure and control methods, in the circuit we can be seen as two six-pulse converter in series, generally work in 2-3 workers Condition, that is, in the 60 repetition period, the two valves and three valves turn on the way of operation. Each 6-pulsed converter has a set of separate commutation transformers, which are connected to the triangular winding on the side of the converter transformer valve side, so that the phase difference of the AC side is 30 degrees, so that good harmonic characteristics are obtained. Simplify the DC and DC side of the filter device, the simulation set the specific parameters shown below. 


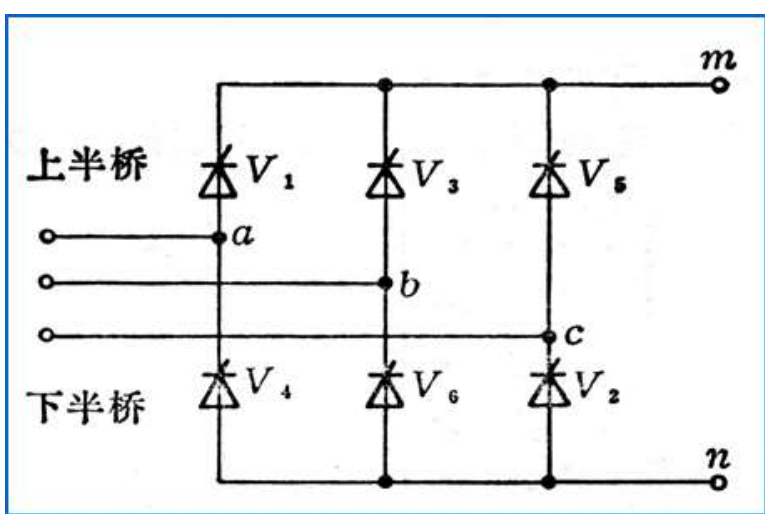

Figure 6 pulsating rectifier structure diagram

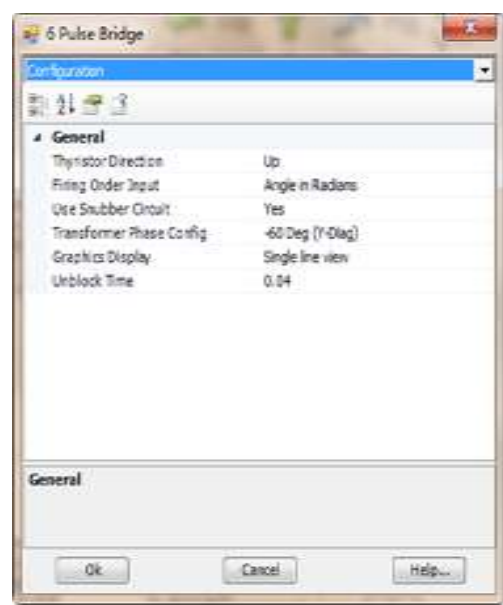

Figure 7 Corresponding parameters

At the same time combined with the reference we learnedSo we calculate the corresponding rectified DC voltage.

Table 1 Explanations of the formula parameters

\begin{tabular}{c|c}
\hline Formula parameters & Related description \\
\hline & Rectifier converter side no - load DC \\
voltage \\
Rectifier valve side rated voltage \\
The positive pressure of the converter \\
valve is $0.3 \mathrm{kV}$ \\
Rated voltage rating \\
& $\begin{array}{c}\text { Six pulse converter number, here is } 2 \\
\text { Rated taper position perceptual pressure } \\
\text { drop per unit value } \\
\text { Rectifier side trigger angle }\end{array}$ \\
\hline
\end{tabular}

Transmission Line. As for the transmission wire modeling is relatively simple, we will wire the corresponding parameters as a number of electrical components. At the same time taking into account the application of flat-wave reactor and DC filter, we add large inductance and grounding capacitance. In summary, in this modeling, we select the electrical model shown below, which is obtained from the relevant reference,

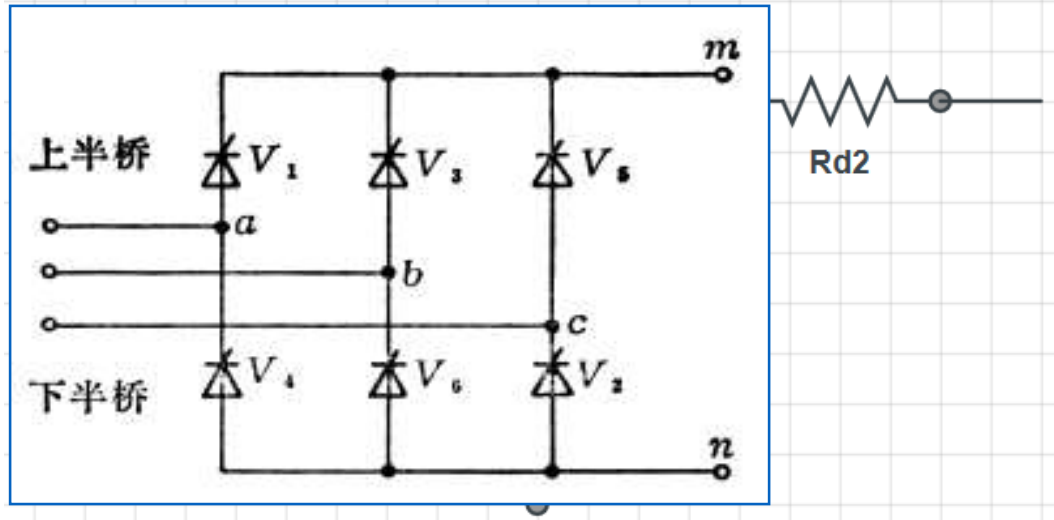

Figure 8. Transmission line structure diagram 


\section{PSCAD Simulation and its Brief Analysis}

Introduction to PSCAD. 1976 by Dennis Dr. Woodford successfully developed and tested the first edition of EMTDC Manitoba Hydro in Canada, for a powerful simulation as an effective means for the Nelson River HVDC project, PSCAD is as user Interface, the role is to facilitate the user to use EMTDC power system analysis and simulation. Since then, the program has been continuously upgraded and has been widely used in many types of simulations of power systems, including exchange studies, lightning overvoltage and power electronics research. With the continuous development of HVDC technology, we need flexible and convenient at the same time can be more accurate simulation software used in the simulation, so we in the next discussion, mainly on the PSCAD simulation.

Simulation of Single - pole HVDC Transmission System Based on PSVCAD. In summary, we use the PSCAD single-pole two-terminal HVDC system architecture, through a certain parameter modification and adjustment, and control structure changes, the corresponding components into the simulation line, to achieve the traditional high-voltage DC transmission of the classic model simulation analysis, the specific circuit as shown Finally, we get the rectifier side and the inverter side current voltage corresponding to the result.

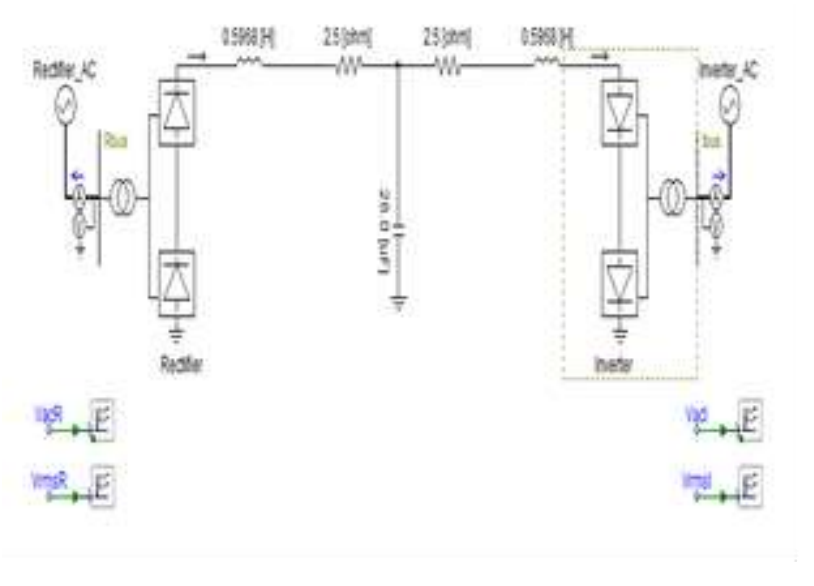

Figure 9. The general framework of PSCAD simulation

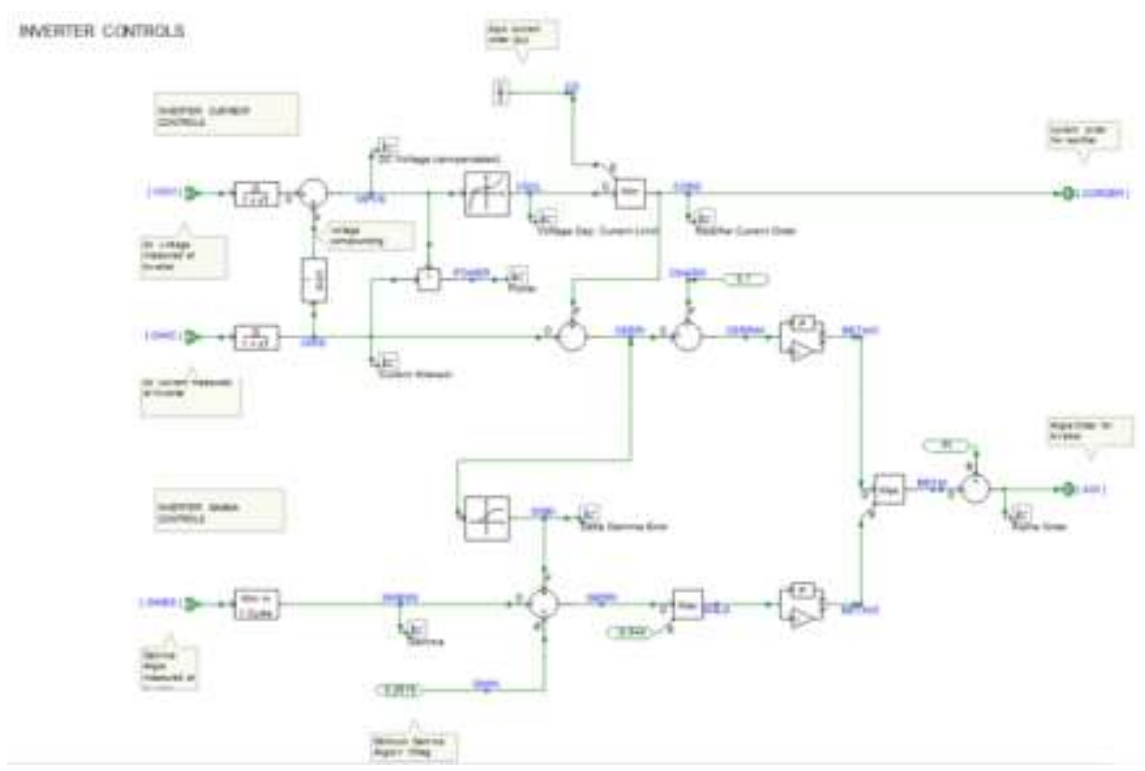

Figure 10. Control unit settings of PSCAD 


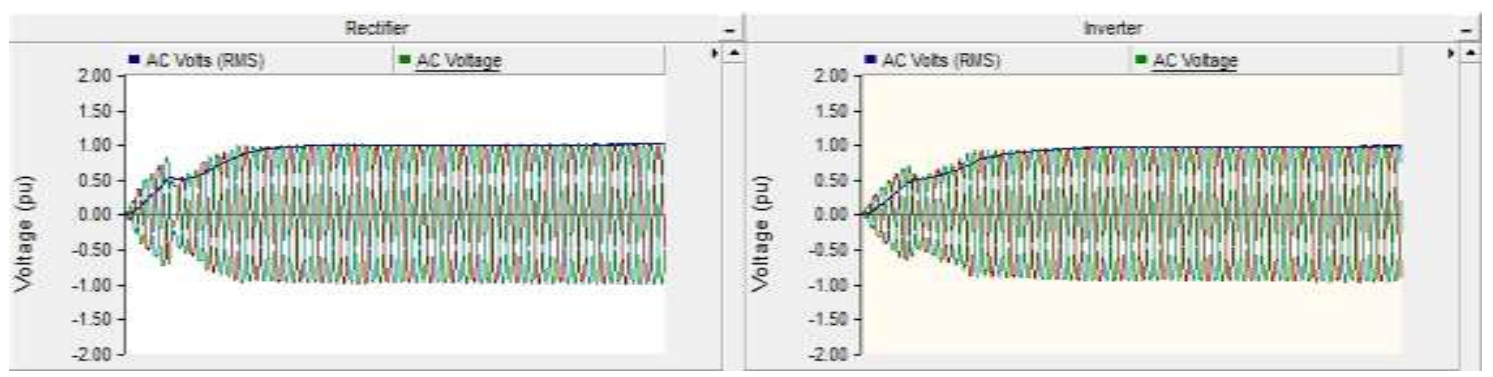

Figure 11. Rectifier and Inverter side AC voltage waveform

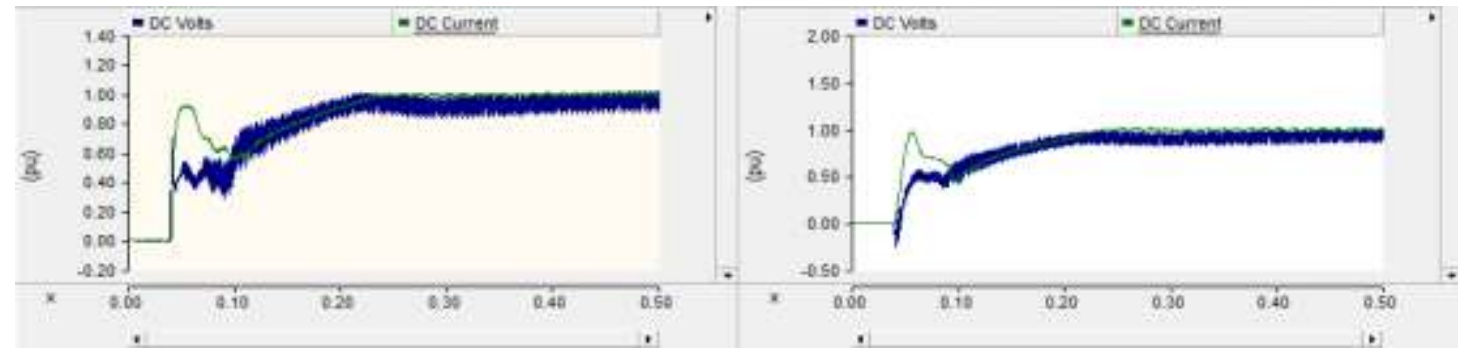

Figure 12. Rectifier and Inverter side DC voltage and current waveform

For the analysis of the waveform, we can get the simulation time of $0.5 \mathrm{~s}$, the simulation step is $10 \mathrm{~s}$, when the time is $0.04 \mathrm{~s}$, the control system of the device is unlocked, the system starts to start, the rectifier side and the inverter side current and voltage waveform similar, But by contrast, the rectifier side of the voltage and current fluctuations more intense and frequent, and after filtering and other processing after the inverter output voltage and current is more smooth and stable. Under the action of the control system, the device undergoes a transient start of a DC voltage rise from zero to zero, after several fluctuations, and finally the smooth operation near the rated value. For the current, in the transient value of the arrival of the stability of the process, the first start will reach a large value instantly, then quickly fall back to 1.0 per unit value near the value, and ultimately with the rise in voltage and steadily reached a stable value.

Then we have a certain numerical calculation of the simulation results, through the waveform analysis we get overshoot,

$$
\delta \%=[\mathrm{Y}(\mathrm{tp})-\mathrm{Y}(\infty)] / \mathrm{Y}(\infty) \times 100 \%
$$
below

As well as its stabilization time per unit value and the triggering angle on both sides, as shown

Table 2 Simulation calculation process parameters

\begin{tabular}{c|c}
\hline Parameter & Results of simulation \\
\hline overshoot & 0.0052 \\
Rectifier stable DC voltage per unit value & 0.892995 \\
Inverter stable DC voltage per unit value & 0.871574 \\
Trigger angle on both sides & $0.31 / 2.56$ \\
\hline
\end{tabular}

Fault Simulation of Single - pole HVDC Transmission System Based on. Through the above process of system startup simulation, we have a certain understanding of it, then we will continue to use PSCAD to its ground short circuit fault simulation analysis. We choose the corresponding fault model, and set the fault parameters, the final structure shown in the figure.

The control unit remains essentially unchanged, and we get the corresponding simulation results, 


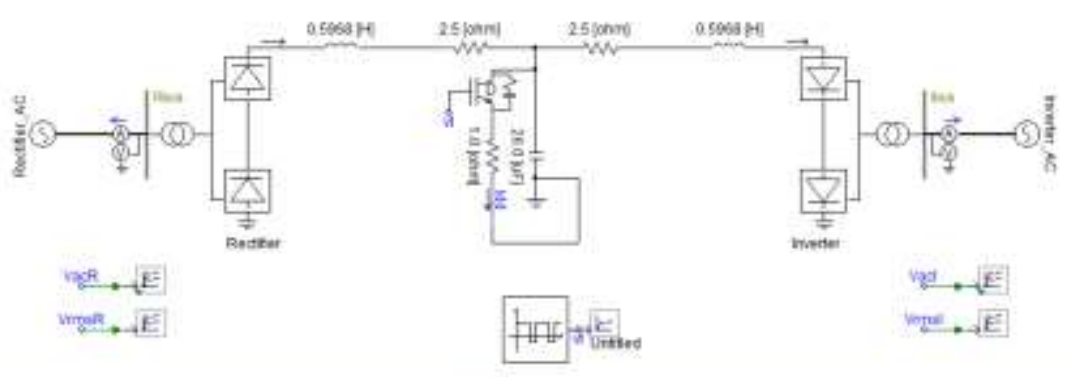

Figure 13. The General Framework of PSCAD Simulation in Short Circuit

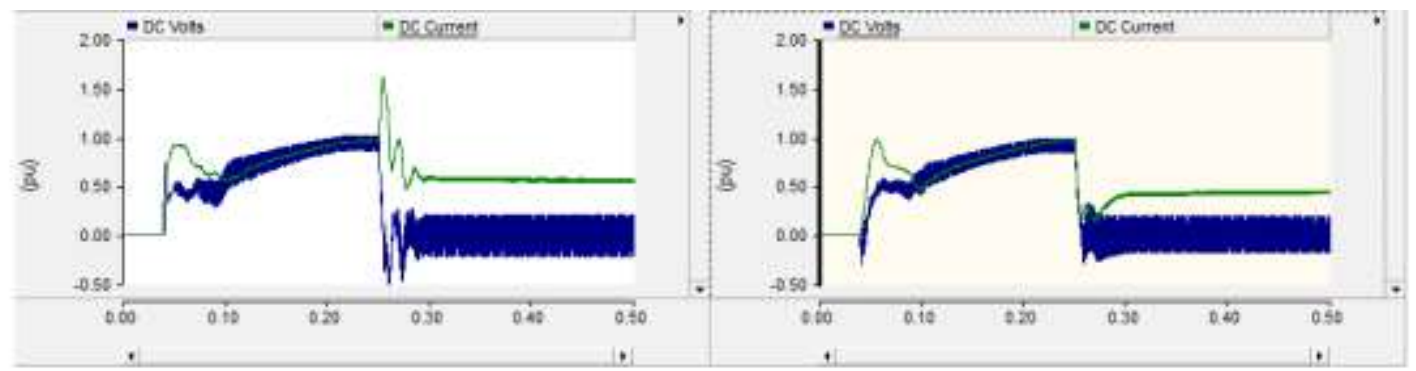

Figure 14. Rectifier and Inverter side DC voltage and current waveform

We can get the analysis of the waveform, the simulation time is $0.5 \mathrm{~s}$, the simulation step is $10 \mathrm{~s}$, the time is $0.04 \mathrm{~s}$, the control system of the device is unlocked, the system starts to start, then starts within $0.25 \mathrm{~s}$. And in the time of $0.25 \mathrm{~s}$, the DC transmission of single-phase ground short-circuit fault, resulting in a sharp decline in voltage on both sides after $0.02 \mathrm{~s}$ or so, because the capacitor inductance charge and discharge and other reasons, the final fluctuations in the vicinity of 0 .And for the current, the two are not the same, the rectifier current first appeared over the current once the value of 1.7 per unit value, this time very easy to burn or break through the line, there is a greater security risks, the need to increase protection. And finally through a week around the stability of 0.5 per unit value in the vicinity, and in the inverter side of the current is a sharp decline in the final $0.3 \mathrm{~s}$ tends to be stable.

\section{Conclusion}

We have analyzed the relevant electrical equipment involved in the operation of HVDC, established the relevant model according to its different electrical characteristics, and adjusted the parameters according to the experience and reference. Especially for the DC transmission converter switching characteristics and commutation process we have a detailed modeling. At the same time, in order to more fully understand the actual operation of the characteristics of high-voltage DC engineering, we use a more flexible and effective simulation software PSCAD simulation. Through the assumption of normal start and short circuit fault, we get the rectification side and the inverter current and voltage waveform, describe the relevant process, and carry on the analysis and calculation.

\section{Reference}

[1] Su Feng Research on Modeling of HVDC Transmission Control System Based on PSCAD [D] North China Electric Power University

[2] Hui Hui Simulation Modeling of HVDC Transmission System [D] North China Electric Power University

[3] Qiu Hang, Wu Wenxuan, Cai Jindin, Huang Ting, Jiang Wei Modeling and Simulation Analysis of UHV DC Transmission [J] Electric Power and Electrical Engineering

[4] Zhang Bihua, Zhou Yunhai, Xiong Wei,Huang Li, Gong Haihua, Wang Yuxi Research on 
Modeling of HVDC Transmission[J] Journal of the Three Gorges University

[5] Zheng Xiaodong, Tai Nengling, Yang Guangliang, Tu Qi Modeling and Simulation of UHVDC Transmission System[J] Electric Power Automation Equipment

[6] Chen Ming, Xiang Yanhui,Guo qianwen, Zheng Tao, Xiao Yexiang, Quan Jiangtao, Ruan Ling Modeling and Simulation of Tianguang $\pm 500 \mathrm{kV}$ HVDC Transmission System[J] Electric Power 